\title{
USABILITY AND PLAYABILITY ISSUES FOR ARQUAKE
}

\author{
Bruce Thomas, Nicholas Krul, Benjamin Close and Wayne Piekarski \\ University of South Australia
}

\begin{abstract}
This paper presents a set of informal studies into the usability and playability of our ARQuake game. ARQuake is an outdoor Augmented Reality version of a first person perspective desktop application. One area of investigation was comparing playing the game along different points of Milgram's RealityVirtuality Continuum: Virtual Reality, Augmented Virtuality, and Augmented Reality. The paper reports on the feedback from a set of users operating ARQuake in an outdoor setting.
\end{abstract}

Key words: Augmented Reality, Wearable Computers, and Outdoor Computer Games

\section{Introduction}

We are investigating placing users in a first person perspective view of virtual world (1), such as games, architectural design viewers (2), geographic information systems and medical applications (3) outdoors, displaying their relevant information via Augmented Reality (AR). In particular we consider the game Quake (4). As with other researchers (5), we wish to place these applications in a spatial context with the physical world, which we achieve by employing our wearable computer system Tinmith (6). Tinmith is a context-aware wearable computer system, allowing applications to sense the position of the user's body and the orientation of the user's head. This paper presents an overview of the ARQuake game and usability and playability issues for ARQuake.

In the ARQuake application, the physical world is modelled as a Quake 3D graphical model. The AR information (monsters, weapons, objects of interest) is displayed in spatial context with the physical world. The Quake

The original version of this chapter was revised: The copyright line was incorrect. This has been corrected. The Erratum to this chapter is available at DOI: 10.1007/978-0-387-35660-0_65 
model of the physical world (walls, ceiling, floors) is not shown to the user: the see-through display allows the user to see the actual wall, ceilings and floors which ARQuake need only model internally. Coincidence of the actual and virtual structures is key to the game; the AR application models the existing physical outdoor structures, and so omission of their rendered image from the display becomes in effect one of our rendering techniques.

ARQuake is a first-person perspective application with the following attributes: 1) The application us situated in the physical world. 2) The point of view, which the application shows to the user, is completely determined by the position and orientation of the user's head. 3) Relevant information is displayed as augmented reality via a head-mounted see-through display. 4) The user is mobile and able to walk through the information space. 5) The user interface additionally requires only a simple hand-held button device.

\section{The ARQuake game}

Quake (4) is a first-person shoot 'em up game, with the user interface based around a single, first-person perspective screen. The goal of ARQuake is to bring the intuitive nature of VR/AR interfaces into an outdoor game. A user first dons the wearable computer on their back, places the head mounted display (HMD) on their head, and holds a simple two-button toy gun input device. The user then performs a simple calibration exercise to align the HMD with their eyes, and then they start playing the game. All of the keyboard and mouse controls have been replaced with position/orientation information and the toy gun controller. As previously mentioned, the user's own movement determines the rate and direction of game movement.

Once the system is up and running, the user moves through the level by physically walking and changes view by looking around. The user views the game and the physical world through the HMD. The bottom portion of the screen is a status bar containing information about armour, health, ammunition and weapon type. The majority of the screen is reserved for the $\mathrm{AR}$ images of monsters and game objects. Because the user's movement is limited to physical walking speeds, monsters are designed to be relatively easy to destroy and do not inflict extreme damage on the user with their first attack. The monsters' skin colour and texture are changed to make them easier to see and distinguish from the physical world.

In the original Quake, certain actions are performed by the user being in a close proximity to a location in a Quake level. We have retained most of those actions. Virtual Quake doors open when the user attempts to walk through them. Users pick up objects as in the original Quake by walking over them. Standing in or moving through predetermined locations triggers 
traps. Actions that are not easily reflected in the physical world are removed from the game, such as secret and locked doors. The tracking of the user's position and orientation of the user's head handles the majority of the interaction. The only other interactions for the user to perform are to shoot or change the current weapon. We employ a two-button (thumb button and index finger button) toy gun as a physical input device for these actions. The thumb button is used to change weapons, and the index finger button fires the current weapon. The direction the weapon fires is the centre of the current view of the HMD.

The Tinmith wearable computer system hardware is mounted on a rigid backpack, see Figure 1. Processing is performed by a Toshiba 320CDS notebook (Pentium-233, $64 \mathrm{Mb}$ RAM) running the freely available Linux operating system. Connected to the laptop are a Precision Navigation TCM280 digital compass for orientation information and a Garmin 12XL GPS receiver for positioning. The HMD is a NTSC Sony Glasstron.

\section{The Informal Experiment}

To gain a broad understanding of the visual effects and playability issues for ARQuake, we implemented five simple Quake worlds to test out different effects. The visual effects fall into three major categories on Milgram's Reality-Virtuality Continuum (7): Virtual Reality, Augmented Virtuality, and Augmented Reality. The Virtual Reality mode of operation is presented as Quake levels being modelled with full textures on all walls and surfaces. The user is able to move around the physical world, but would not use any cues of the physical world for navigation. The Augmented Reality mode only renders the game's pieces and monsters as described before. The Augmented Virtuality mode places large walls and surfaces in the game, but the physical world is also viewed alongside these. An example of this Augmented Virtuality is a set of rooms placed in the middle of a large open space between buildings. The user sometimes interacts in an Augmented Reality setting and sometimes in a Virtual Reality setting, and the switch between them is seamless to the user.

User's were taken outside and asked to play short segments of the game containing these different visual effects. These sessions lasted for about an hour, and a researcher was present during these trials. The researcher recorded the comments via paper and pencil, and helped the user with the operation of the game. The sessions included showing the users the three different categories: Virtual Reality, Augmented Virtuality, and Augmented Reality. We experimented with rooms and corridors of different sizes. We 
looked at different textures and placement of objects at varying heights and sizes.

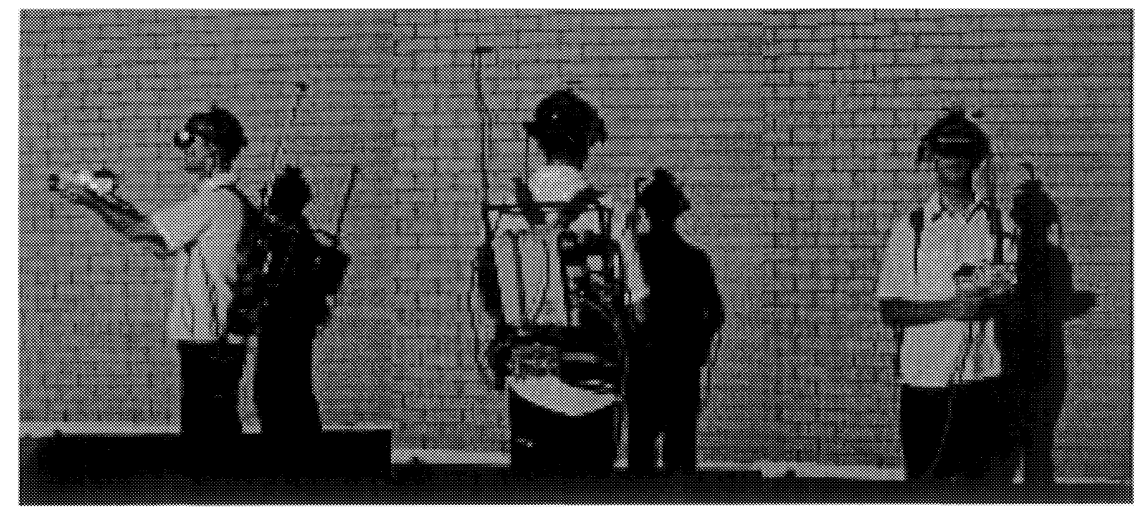

Figure 1. Wearable Computer System

\section{User Comments}

We recorded the users' comments while they were operating ARQuake outdoors. The comments are grouped in the following categories: field of view, tracking, presence, and movement. We present here a condensed version of the comments recorded.

\subsection{Field of view}

The field of view (FOV) of the HMD used in the experiment was 24 degrees horizontal, and this narrow FOV necessitated excessive head turning by the users. The FOV of 24 was thought to be "extremely" narrow. A user asked to change the FOV to 90 degrees after a short while, and they commented that this felt much better. They did think it might cause problems within an AR environment, because the FOV of the graphical images would not overlap the FOV of the physical world. They commented this could be somehow incorporated into the game in some fashion. A different user stated they liked the game, but wished the FOV was much closer to their own. A second user asked the FOV of the graphics be changed to 120 degrees. They stated the experience was "weird."; stating difference in parallax between the physical world and the virtual world was very uncomfortable. They did not notice it at a FOV of 90 degrees, but at a FOV of 120 degrees the effect was "bad." A second feature they found was the 
amount of "zoom" that a narrow FOV involves is misleading. They felt the apparent distance was correct at a FOV of 90 degrees. (This is probably due to the Quake graphics are designed to be operated at a FOV of 90 degrees). With a narrow field of view, objects appeared closer then they really were. A comment was made the narrow field of vision of the glasses strained the user's eyes. They stated better visual equipment would help. They went on to comment that a better positioning system would solve the problem, as the objects jump out the FOV due to tracking errors.

\subsection{Tracking}

Our system used a standard 12 channel GPS unit, and it introduced tracking errors into the game. A user remarked that the graphics were "a little jumpy", and they believed a larger map might be advisable. The users felt the small levels presented were not playable, due to the accuracy of the tracking. The compass also added tracking error, but of an orientation nature. A user thought the compass should be set to tilt forwards slightly when one is looking level. This turned out to be caused by the compass being incorrectly initialised. A small error turned out to be quite noticeable to this user.

\subsection{Presence}

Thick or "chunky" doors and narrow corridors had a number of unpleasant characteristics. They made the user's feel claustrophobic, "like there isn't enough space to get through the door." The "chunkiness" caused trouble while going through the door, and narrow corridors required "too much time" aligning oneself to the corridor. Figure 2 depicts two examples of narrow corridors.

While the user was in the Virtual Reality mode, the contrast of the HMD was set its maximum value. The effect for the user was a black wall appeared solid and not transparent. The users did not like darkened rooms. Large black areas made the users feel lost, and even small dark areas, because of the narrow FOV. They preferred the walls and objects well lit.

The use of virtual floors in the Augmented Virtuality worlds raised a number of comments. In the Augmented Virtuality mode, the contrast of the HMD was set to allow the user to clearly see both the physical world when viewing a black wall and to occlude the physical world with a brightly textured wall. One user stated they did not like virtual floors, because they did not like seeing the ground twice. One of the Quake worlds incorporated light coloured floors with dark (therefore transparent) paths. This caused an "inverted world" look, where the solid object (the path) was see-through. It 
seemed to make the user feel like they are in the virtual world, with a minor sense of presence in the physical world.
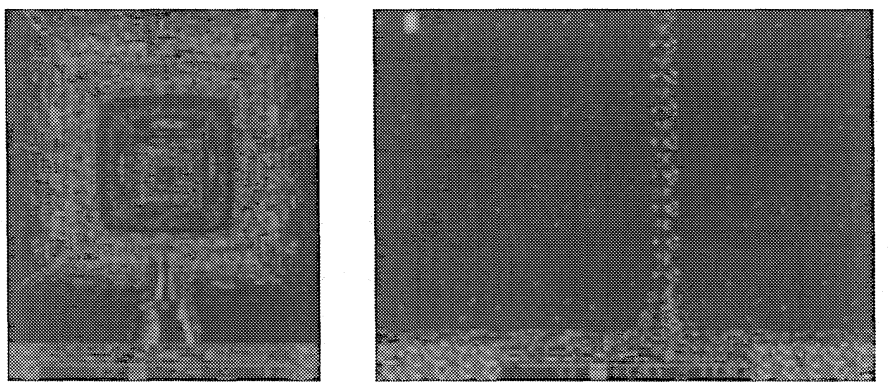

Figure 2. Two Examples of Narrow Corridors

The use of floating objects worked well in the different modes of the Quake world, "they looked like they belonged - in the game world." One user stated they simply accepted them as part of the game, and might have been because they were obviously not in the real world. One user stated, he was "sucked" into the virtual world and while he was mindful of the real world, he was mostly in the virtual one. Figure 3 depicts two different floating objects. One particular graphic effect the users commented on with the flames. They provided comments like, "the fire in Quake looked pretty good" and "it looked genuinely like fire."
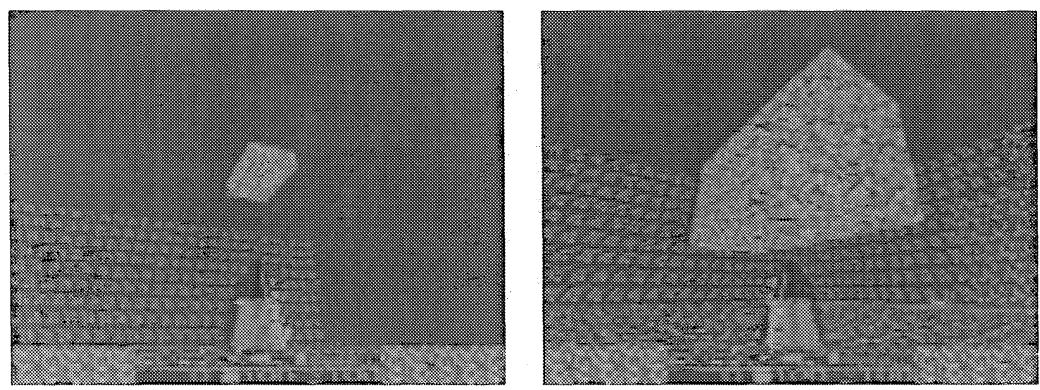

Figure 3. Two Examples of Floating Objects

Two different sky backgrounds were investigated, purple and blue. The purple sky jarred a user's eyes; it did not match with the physical sky. The blue sky was deemed to be better suited to the game, as it blends in with the physical sky to provide the user with effect of only one sky. This was deemed to be acceptable, provided that one was looking at the sky. If buildings were overlapped by a virtual sky, it made the game world less believable. The user's felt the sky was something that had to be transparent. 
A number of users stated the virtual shadows did not look like real shadows. In the Augmented Virtuality mode they looked more like the real world was leaking into the virtual one. In the Virtual Reality and Augmented Virtuality modes found shadows quite annoying. At some points it made the game unplayable. One user stated the did not like seeing shadows in the virtual world and knowing they were not there in the physical world, as you could see they were not projected. Figure 4 depicts two examples of textures that would allow "leakage" from the physical world into the virtual world.
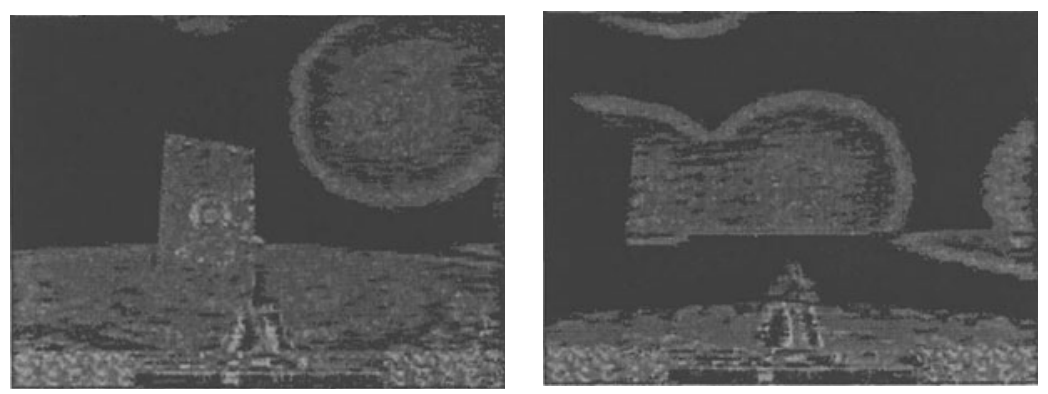

Figure 4. . Two Examples Leakage - the real world could be seen through the dark shadows

The users were able to walk through the virtual walls. One user stated "Walking through walls, while being an interesting idea, has the problem that, when you get so close to a wall, ... you can see the texture of the wall to the pixel level. It looked solid, but I wasn't worried about walking through it because I knew that there was a room on the other side. Strangely enough, I didn't want to go out the other side because I knew that the map didn't extend there."

One user noticed at one point they were physically walking up a hill, but the game depicted flat ground. They stated it seemed to feel, while unexpected, easier to walk up because it looked flat.

\subsection{Movement}

User's experienced difficulties picking objects up. They felt they were "tantalizingly" within arms reach, but sometimes they could not be picked up. This problem became quite frustrating for some of the users. We believe this a problem with the GPS resolution and the incorrect FOV.

Opening and closing doors was not difficult for the users, but one user was killed off in the game when a door opened and closed without notice. This unexpected action caused some frustration to the user. 
We experimented with flying monsters. The user required the correct weapon and the correct strategy to deal with them, or the monster would kill the user very quickly. We believe these forms of monsters could make for an interesting game - testing the learning curve on "solving" all of the puzzles.

\section{Conclusion}

In conclusion, we found a number issues for constructing the virtual worlds of ARQuake more usable and playable. We experimented with a number of different field of view (FOV) settings. Setting the FOV to the same as the HMD corrected for the registration of the graphical images, but cause depth perception problems. This FOV problem requires further investigation. Small errors in the tilt of compass turned out to be quite noticeable. The standard shape of doors and corridors in the Quake worlds are not well suited to ARQuake. ARQuake requires wider corridors and doors that are not so "chunky." The world feels better to the user if it is brightly lit. In the Augmented Virtuality and Augmented Reality modes, very dark and black colours should be avoided. These colours give the illusion the physical world is "leaking" into the virtual world. Floating objects work quite well in ARQuake. The use of a sky colour should be avoided. Users did not like the use of shadows in the virtual worlds. Even with these comments, the users overall enjoyed ARQuake.

\section{References}

1. Thomas BH, Close B, Donoghue J, Squires J, DeBondi P and Piekarski W. First person indoor/outdoor augmented reality application: ARQuake. Personal and Ubiquitous Computing 6, 2002.

2. Brooks FP. Walkthrough -- A Dynamic Graphics System for Simulating Virtual Buildings. Workshop on Interactive 3D Graphics, 1986.

3. State A, Livingston MA, Garrett WF, Hirota G, Whitton MC, Pisano ED and Fuchs H. Technologies for Augmented-Reality Systems: Realizing Ultrasound-Guided Needle Biopsies. Proceedings of SIGGRAPH 1996, New Orleans, Louisiana. ACM, 1996, p. 429438.

4. idSoftware. Quake. http://www.idsoftware.com/

5. Hollerer T, Feiner S and Pavlik J. Situated Documentaries: Embbeding Multimedia Presentations in the Real World. In: 3nd International Symposium on Wearable Computers. San Francisco, CA, 1999, p. 79-86.

6. Piekarski W and Thomas B. Tinmith-evo5: An Architecture for Supporting Mobile Augmented Reality Environments. Second IEEE and ACM International Symposium on Augmented Reality, New York, NY. IEEE, 2001.

7 Milgram P, Takemura H, Utsumi A and Kishino F. Augmented Reality: A Class of Displays on the Reality Virtuality Continuum. Telemanipulator and Telepresence Technologies. SPIE, 1994. 